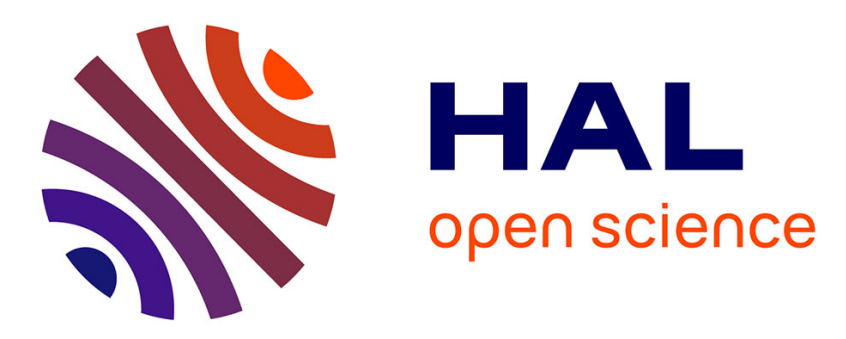

\title{
Applications métallurgiques des techniques de microanalyse par l'observation directe de réactions nucléaires
}

\author{
G. Amsel, D. David, G. Béranger, P. Boisot
}

\section{- To cite this version:}

G. Amsel, D. David, G. Béranger, P. Boisot. Applications métallurgiques des techniques de microanalyse par l'observation directe de réactions nucléaires. Revue de Physique Appliquée, 1968,3 (4), pp.373-386. 10.1051/rphysap:0196800304037300 . jpa-00242875

\section{HAL Id: jpa-00242875 https://hal.science/jpa-00242875}

Submitted on 1 Jan 1968

HAL is a multi-disciplinary open access archive for the deposit and dissemination of scientific research documents, whether they are published or not. The documents may come from teaching and research institutions in France or abroad, or from public or private research centers.
L'archive ouverte pluridisciplinaire HAL, est destinée au dépôt et à la diffusion de documents scientifiques de niveau recherche, publiés ou non, émanant des établissements d'enseignement et de recherche français ou étrangers, des laboratoires publics ou privés. 


\title{
APPLIGATIONS MÉTALLURGIQUES DES TEGHNIQUES DE MICROANALYSE PAR L'OBSERVATION DIREGTE DE RÉAGTIONS NUGLÉAIRES
}

\author{
Par G. AMSEL et D. DAVID, \\ Groupe de Physique du Solide de l'École Normale Supérieure, 9, quai Saint-Bernard, Tour 23, Paris, 5e, \\ et G. BÉRANGER et P. BOISOT, \\ Centre de Recherche Métallurgique, École Nationale Supérieure des Mines de Paris.
}

\begin{abstract}
Résumé. - La méthode de microanalyse par réactions nucléaires est brièvement exposée après une comparaison aux autres méthodes de microanalyse, telles que les sondes électronique et ionique, l'analyse par activation, etc. La spécificité et la haute sensibilité de cette technique sont décrites. On passe ensuite en revue diverses applications à la métallurgie, illustrées par des résultats expérimentaux : diffusion de l'oxygène dans le zirconium, autodiffusion de l'oxygène dans la zircone (par $\mathrm{O}^{18}$ ), perturbations de surface induites par divers traitements de polissage, effet d'un recuit sous vide poussé, nitruration de divers métaux dans l'ammoniac chaud, etc. Les résultats présentés portent plus particulièrement sur le dosage des isotopes de l'oxygène et de l'azote, mais l'extension de ces techniques à d'autres éléments légers est évoquée.
\end{abstract}

Abstract. - Microanalysis by means of nuclear reactions is briefly described, and compared with other microanalytical methods such as electron and ion probe, activation analysis, etc. The specific character and high sensitivity of the method are emphasized. A review follows of some applications to metallurgy, illustrated by experimental results : oxygen diffusion in zirconium and self-diffusion in zirconia (using $\mathrm{O}^{18}$ ), perturbations induced by various surface treatments, influence of annealing in high vacuum, nitriding of various metals in hot ammonia, etc. The results shown are chiefly related to the determination of the isotopes of oxygen and nitrogen, but the extension of these techniques to other light elements is also approached.

I. Introduction. - Il existe actuellement de nombreuses méthodes pour aborder le délicat problème du dosage des éléments légers dans une matrice : analyse par activation, spectroscopies infrarouge et hertzienne, microanalyse à la sonde ionique et à la sonde électronique, spectrographie de masse.

Le microanalyseur à sonde électronique de Castaing [1], [2], permet actuellement la détection et l'analyse des éléments légers tels que l'oxygène, le carbone, l'azote. Mais il ne permet pas de faire les distinctions entre deux isotopes, ce qui élimine toute expérience de marquage permettant d'atteindre, par exemple, les mécanismes de croissance des couches d'oxyde. De plus, la mauvaise connaissance des coefficients d'absorption par la matrice des rayonnements mis en jeu ne permet pas de faire les corrections appropriées pour obtenir un dosage précis dans le cas des éléments légers.

La technique d'analyse par activation [3], [4], [5], fait appel à des procédés utilisés couramment avec

(1) Compte rendu de travaux exécutés dans le cadre de la R.C.P., no 157, du C.N.R.S., avec le soutien de la D.R.M.E. les traceurs radioactifs et a été fréquemment employée comme alternative pratique à l'analyse par spectromètre de masse. Cependant, au cours de l'irradiation, d'autres noyaux que ceux de l'élément étudié peuvent être activés, ce qui pose des problèmes d'identification parfois difficiles à résoudre. Aussi, pour obtenir une bonne précision, est-on amené le plus souvent à effectuer postérieurement à l'irradiation des séparations chimiques, ce qui implique la destruction de l'échantillon. D'autre part, il est nécessaire que le noyau activé produit ait une période suffisamment longue pour permettre les comptages et obtenir une bonne précision.

Le dosage par résonance magnétique nucléaire est assez peu utilisé car il ne peut s'appliquer à des éléments dont le spin est nul. En particulier, il est impossible de doser les isotopes $\mathrm{O}^{16}$ ou $\mathrm{O}^{18}$ par cette méthode qui, cependant, s'applique à l'isotope $\mathrm{O}^{17}$ mais avec une faible sensibilité, bien qu'un enrichissement élevé en $\mathrm{O}^{17}$ soit maintenant possible (environ $10 \%$ pour l'eau).

Dans quelques cas bien particuliers, la spectrométrie infrarouge peut être employée pour l'analyse isotopique de $\mathrm{O}^{18}$ dans les couches superficielles, comme 
cela a été fait dans le cas de l'oxydation du silicium [6].

La microanalyse par émission ionique secondaire [7], [8], méthode qui combine la microscopie ionique et la spectrométrie de masse, permet une analyse locale chimique et isotopique. Tous les éléments sont analysables quel que soit leur numéro atomique, et la sensibilité de détection est très élevée. Cependant, le rendement d'ionisation dépend fortement de la structure cristalline du matériau utilisé, ce qui constitue une difficulté importante pour l'analyse des solides polycristallins. Cette difficulté est cependant moins sérieuse si on mesure seulement la composition isotopique relative, ou si l'on dose les éléments légers dans des échantillons monocristallins. En outre, l'interprétation quantitative des résultats fait encore l'objet d'études fondamentales, ce qui limite actuellement les applications possibles. Il n'en reste pas moins que cette méthode décrite en détail par G. Slodzian dans le même numéro de ce journal est d'une très grande puissance et peut constituer une alternative intéressante aux méthodes décrites brièvement dans cet article. Cet article ainsi que celui de Slodzian permettent de se faire une idée des domaines d'utilisation où les deux méthodes se recouvrent ou au contraire se complètent.

Ces différentes méthodes ne permettent donc pas toujours de détecter les éléments légers avec la précision et la sensibilité nécessaires. D'autre part, le dosage quantitatif de faibles traces d'éléments dans le cas des réactions superficielles, en particulier des phénomènes de diffusion, de corrosion, d'oxydation qui jouent un rôle important dans le comportement des métaux et des semiconducteurs, est souvent difficile. Ces remarques ont amené Amsel et ses collaborateurs à mettre au point une méthode de microanalyse fondée sur l'observation directe des réactions nucléaires, et qui permet de doser avec une bonne précision et une haute sensibilité les éléments présents au voisinage de l'échantillon à étudier [9], [10], [11], [12]. L'observation directe des produits d'une réaction nucléaire conduit au dosage et à la localisation des noyaux provoquant la réaction. Précisons qu'en général avec cette méthode non destructive la résolution est de l'ordre du dixième de micron; dans certains cas même, par exemple le dosage de l'oxygène 18 , l'utilisation d'une résonance nucléaire permet d'atteindre une résolution voisine de $100 \AA$ [9]. La précision de la mesure des concentrations par cette méthode est dans le cas de l'oxygène meilleure que $1 \%$, sans dépasser des durées d'expérimentation de quelques minutes dans la plupart des cas; il est possible de mettre en évidence des quantités d'oxygène de $10^{-12} \mathrm{~g}$ par $\mathrm{cm}^{2}$, ce qui correspond à environ $2 \times 10^{14}$ atomes par $\mathrm{cm}^{2}[11]$.

Des techniques analogues ont déjà été utilisées par d'autres auteurs. Ainsi Ollerhead et coll. [13] ont utilisé la réaction $\mathrm{O}^{17}\left(\mathrm{He}^{3}, \mathrm{He}^{4}\right) \mathrm{O}^{16}$ pour doser l'isotope 17 de l'oxygène dans la zircone. Cox et Roy [14], à l'aide de la même réaction, ont déterminé des profils de concentration de l'oxygène 17 dans la zircone en fonction de la profondeur. Möller et Starfelt [15], quant à eux, ont dosé le fluor dans le zircaloy en utilisant la réaction $\mathrm{F}^{\mathbf{1 9}}(\mathrm{p}, \alpha \gamma) \mathrm{O}^{\mathbf{1 6}}$.

Toutefois, en ce qui concerne l'oxygène, Ollerhead et coll. [13] ainsi que Cox et Roy [14] n'ont pas étudié les réactions concernant les isotopes $\mathrm{O}^{16}$ et $\mathrm{O}^{18}$ qui sont les deux isotopes les plus fréquents. Par contre, Amsel et ses collaborateurs ont plus particulièrement travaillé sur ces deux isotopes en systématisant la méthode (utilisation de références standard par exemple) et étendu celle-ci à d'autres éléments. Un article tout à fait général [16] sur l'analyse par observation des réactions nucléaires étant actuellement en préparation, nous ne présentons ici que les résultats de plusieurs années d'expériences obtenus, dans le domaine de la métallurgie, à l'aide de cette méthode appliquée plus particulièrement au dosage de l'oxygène et de l'azote.

II. Description de la méthode. - Nous allons, dans ce qui suit, décrire essentiellement les applications de la méthode fondée sur l'enregistrement et l'interprétation des spectres d'énergie des particules émises au cours des réactions nucléaires provoquées par le bombardement de la cible à étudier par un faisceau de deutons ou de protons, la méthode basée sur l'utilisation des résonances (cas du dosage de l'oxygène 18 par exemple) n'étant que rapidement évoquée. Cette méthode a été plus particulièrement appliquée pour doser l'oxygène, l'azote et le carbone; le tableau I rassemble des réactions possibles dans le cas de ces trois éléments. Mais elle est également applicable à de nombreux autres éléments comme le soufre, le fluor, le silicium, le deutérium.

L'énergie des particules détectées est spécifique du noyau bombardé, ce qui permet de déterminer la nature des éléments présents au voisinage de la surface de l'échantillon sur une profondeur de quelques microns (2 à $3 \mu \mathrm{m}$ ), de les localiser en profondeur par

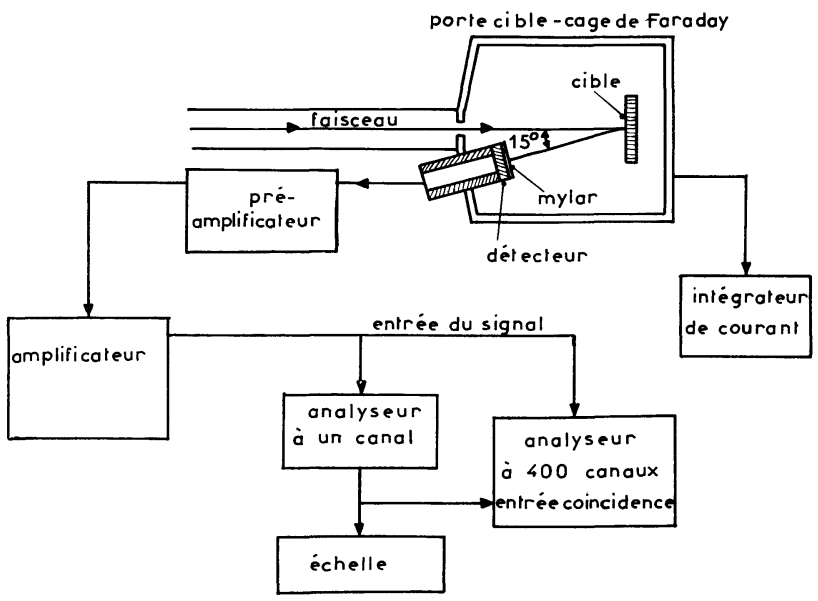

FIG. 1.

Schéma de principe de l'appareillage et géométrie. 
TABLEAU I

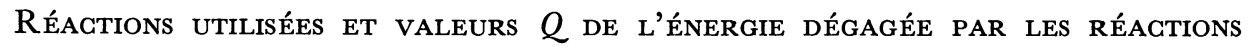

Oxygène

Azote $\left\{\begin{array}{l}\mathrm{N} \\ \mathrm{N}\end{array}\right.$

$\begin{array}{cc} & \mathrm{C}^{12}(\mathrm{~d}, \mathrm{p}) \mathrm{C}^{13} \\ \text { Carbone } \quad \mathrm{G}^{12} & Q=2,72 \mathrm{MeV}\end{array}$$$
\mathrm{N}^{14}(\mathrm{~d}, \alpha) \mathrm{C}^{12}
$$$$
Q=13,6 \mathrm{MeV}
$$$$
\mathrm{N}^{15}(\mathrm{p}, \alpha) \mathrm{C}^{12}
$$$$
Q=5 \mathrm{MeV}
$$$$
\mathrm{G}^{12}(\mathrm{~d}, \mathrm{p}) \mathrm{C}^{13}
$$$$
Q=2,72 \mathrm{MeV}
$$

$$
\begin{array}{cc}
\mathrm{O}^{18}(\mathrm{p}, \alpha) \mathrm{N}^{15} & \mathrm{O}^{18}(\mathrm{~d}, \alpha) \mathrm{N}^{16} \\
Q=3,97 \mathrm{MeV} & Q=4,24 \mathrm{MeV} \\
\mathrm{O}^{16}(\mathrm{~d}, \alpha) \mathrm{N}^{14} & \mathrm{O}^{16}(\mathrm{~d}, \mathrm{p}) \mathrm{O}^{17} \\
- & - \\
Q=3,116 \mathrm{MeV} & Q_{0}=1,919 \mathrm{MeV} \\
& Q_{1}=1,048 \mathrm{MeV}
\end{array}
$$

rapport à cette surface et, par comparaison avec des étalons, de les doser quantitativement.

Nous allons décrire cette méthode dans le cas particulier du dosage de l'oxygène 16 à l'aide de la réaction $\mathrm{O}^{16}(\mathrm{~d}, \mathrm{p}) \mathrm{O}^{17^{*}}$, réaction qu'il est préférable d'utiliser car elle présente une section efficace très grande et fournit un pic de protons bien isolé, sauf en présence d'azote. Notons que tout ce qui va suivre peut également se transposer au cas du dosage d'autres éléments légers.

Considérons un deuton incident qui arrive à la surface de l'échantillon ( $f g$. . 1); il pénètre dans le matériau et subit une perte d'énergie au cours de sa traversée dans la matrice jusqu'à la profondeur $x$ où la réaction nucléaire $\mathrm{O}^{16}(\mathrm{~d}, \mathrm{p}) \mathrm{O}^{17^{*}}$ a lieu. L'énergie $E_{\mathrm{p}}$ du proton détecté, émis par la réaction à cette profondeur dépend, si l'on suppose le milieu homogène, des paramètres suivants :

- de l'énergie du deuton incident,

- de la perte d'énergie dans la matière subie par le deuton pour atteindre la profondeur $x$,

- de la perte d'énergie subie par le proton pour ressortir de la matière, le chemin parcouru étant $x /|\cos (\theta)|$ où $\theta$ représente l'angle de détection, l'échantillon étant perpendiculaire au faisceau,$$
\mathrm{O}^{18}(\mathrm{~d}, \mathrm{p}) \mathrm{O}^{19}
$$$$
Q_{0}=1,731 \mathrm{MeV}
$$$$
Q_{1}=1,635 \mathrm{MeV}
$$

$$
\mathrm{N}^{14}(\mathrm{~d}, \mathrm{p}) \mathrm{N}^{15}
$$$$
Q=8,6 \mathrm{MeV}
$$

$Q=8,6 \mathrm{MeV}$

- de la perte d'énergie subie par ce proton lors de la traversée du film mince de matière plastique (Mylar) qui, placé devant le détecteur, absorbe les deutons rétrodiffusés.

Il résulte de ce qui précède que l'énergie d'un proton détecté est d'autant plus faible que la profondeur à laquelle il a été émis est plus grande, pour des deutons incidents d'énergie donnée.

Le nombre de protons ayant l'énergie $E_{\mathrm{p}}(x)$ dépend également de plusieurs facteurs :

- la concentration $C(x)$ en oxygène à la profondeur $x$,

- la section efficace de la réaction $\mathrm{O}^{16}(\mathrm{~d}, \mathrm{p}) \mathrm{O}^{17^{*}}$ en fonction de l'énergie (c'est-à-dire le rendement de réaction des deutons en fonction de leur énergie),

- les caractéristiques du système de détection, en particulier l'angle auquel se fait la détection par rapport au faisceau incident, de l'angle solide surtendu par le détecteur, de la résolution du détecteur et de la fluctuation statistique de la perte d'énergie après la traversée du Mylar,

- la fluctuation statistique de la perte d'énergie des particules lors de la traversée du matériau de la profondeur jusqu'à la surface.

Si les phénomènes de fluctuation étaient négligeables et si de plus la résolution du système de détec- 
tion (détecteur à semiconducteur et amplificateur Ortec couplés à un analyseur Intertechnique à 400 canaux) était parfaite, il y aurait une relation biunivoque entre l'énergie du proton détecté et la profondeur à laquelle il a été émis. Ainsi on pourrait, dans le cas de l'oxygène, déduire directement la courbe de concentration en oxygène dans l'échantillon étudié, du spectre expérimental, en supposant connues les relations parcours-énergie des deutons et des protons dans le métal étudié ainsi que la section efficace de la réaction nucléaire envisagée. On doit considérer en fait que le spectre expérimental enregistré sur l'analyseur multicanaux résulte de la convolution d'un spectre théorique (que l'on obtiendrait si les phénomènes de "straggling » étaient négligeables et si la résolution de la chaîne de détection était parfaite) avec une « fonction d'instrument » qui caractérise la résolution de l'appareillage, les fluctuations dues à la traversée du matériau étudié pouvant être en général considérées comme négligeables par rapport à celles dues à la traversée du Mylar.

L'opération mathématique qui permet de passer du spectre expérimental à la courbe de concentration peut être assez délicate dans certains cas où l'on peut être amené à faire soit une déconvolution du spectre expérimental pour tenir compte de fluctuations statistiques dues à l'appareillage, soit un ajustement du spectre expérimental à des spectres calculés théoriquement pour différents profils de concentration. Cependant, dans la plupart des cas, il est possible de déterminer directement la concentration en fonction de la profondeur sans faire d'hypothèses particulières.

RELAtions PARGours-Énergie. - Nous avons vu qu'il est indispensable de connaître les relations parcours-énergie des particules (deutons, protons...) dans le matériau étudié ainsi qu'à travers le Mylar, pour pouvoir interpréter les spectres expérimentaux. Nous disposons actuellement des tables sur les pouvoirs d'arrêt ou relations parcours-énergie établies par Williamson, Boujot et Picard [18]. Ces tables concernent actuellement la plupart des éléments. Cependant, lorsque nous ne disposions pas de ces tables, nous avons pu calculer les relations parcours-énergie à l'aide d'une méthode d'interpolation logarithmique en fonction du numéro atomique à partir des données relatives à d'autres éléments comme le cuivre et le xénon par exemple [17].

Nous avons pu contrôler la validité de ce raisonnement en comparant les valeurs calculées pour le cuivre par cette méthode d'interpolation linéaire à partir du xénon et du krypton [17]. L'erreur commise sur la relation parcours-énergie est d'environ $5 \%$. Ces résultats sont confirmés par les tables calculées par Williamson [18]. D'autre part, connaissant les pouvoirs d'arrêt relatifs aux protons dans un matériau donné, il est facile d'en déduire les données relatives aux deutons et inversement. On dispose en effet de la relation :

$$
R_{\mathrm{d}}(E)=2 R_{\mathrm{p}}(E / 2)
$$

où $R_{\mathrm{d}}$ et $R_{\mathrm{p}}$ représentent respectivement les parcours des deutons et des protons. Les relations parcoursénergie dans le Mylar ont été établies à partir des données dans l'air [17], l'équivalence en épaisseur ayant été mesurée directement.

Segtion efficace. - La section efficace d'une réaction nucléaire telle que $\mathrm{O}^{16}(\mathrm{~d}, \mathrm{p}) \mathrm{O}^{17^{*}}$ pour un angle d'observation donné est une fonction de l'énergie des particules incidentes : elle caractérise la probabilité de réaction des particules incidentes en fonction de leur énergie. La détermination expérimentale a été faite sur une cible mince d'oxyde de tantale $\mathrm{Ta}_{2} \mathrm{O}_{5}$ préparée par oxydation anodique. On bombarde la cible à l'aide de deutons d'énergie variable et l'on détermine dans les conditions de détection utilisées le nombre de protons émis en fonction de l'énergie des particules. Les résultats obtenus sont représentés sur la figure 2.

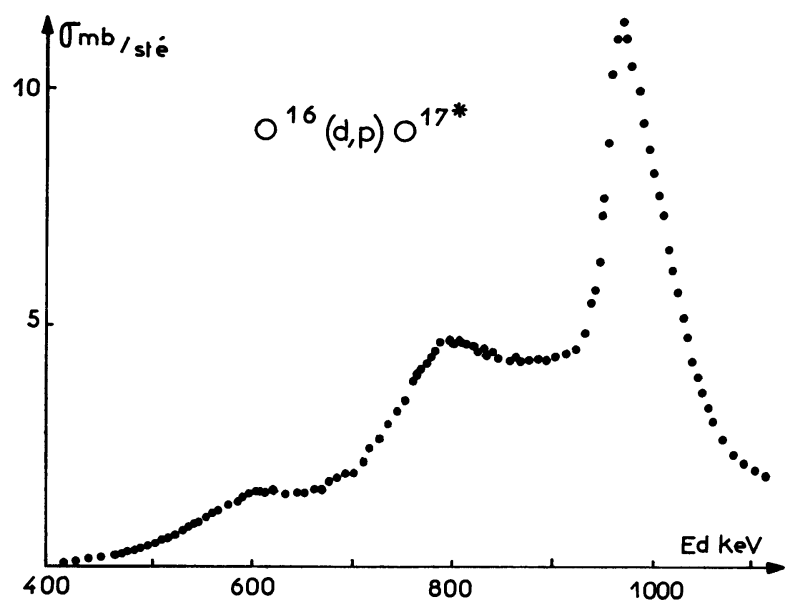

FIG. 2. - Section efficace différentielle à $165^{\circ}$ de la réaction $\mathrm{O}^{16}(\mathrm{~d}, \mathrm{p}) \mathrm{O}^{17^{*}}$.

III. Analyse des résultats. - Comme nous l'avons déjà remarqué précédemment, il y a deux cas à envisager pour l'interprétation des résultats obtenus, à l'aide de cette méthode, suivant que l'on se trouve dans le cas d'une diffusion peu profonde (inférieure à 2 ou $3 \mu \mathrm{m}$ ) ou non.

a) Diffusion peu profonde. - Dans ce cas, un seul spectre permet de déterminer les caractéristiques de diffusion de l'élément à étudier. On compare par détermination de l'écart quadratique moyen les spectres expérimentaux à des spectres calculés pour différents profils de concentration ( fig. 3), comme cela a été fait dans le cas du quartz [12]. En effet, on peut calculer en tenant compte des relations parcoursénergie des particules, de la section efficace de la réaction nucléaire envisagée, des réseaux de spectres correspondant par exemple à des profils de concentration pouvant s'exprimer par une fonction erreur complémentaire de la profondeur, pour diverses va- 


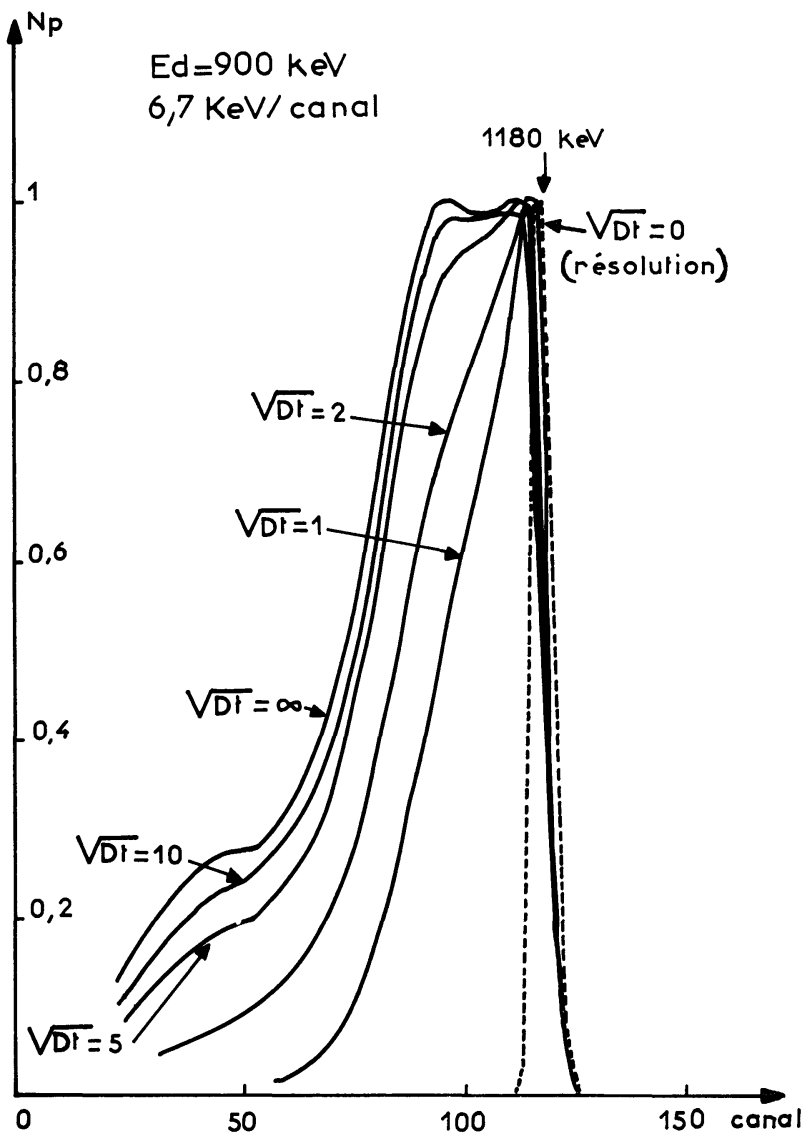

FIG. 3. - Spectres calculés correspondant à différentes valeurs de $\sqrt{D t}$ (en microns) dans l'hypothèse d'une diffusion en fonction erreur dans le zirconium.

leurs du coefficient de diffusion (cas de la figure 3) [21]. Dans certains cas, comme celui d'une couche mince d'oxyde, de nitrure, de siliciure à la surface d'un échantillon, on peut être amené à procéder à la décomposition du spectre enregistré [19], c'est-à-dire séparer les particules provenant des réactions nucléaires avec les atomes contenus dans la couche superficielle de celles provenant de l'oxygène dissous dans le métal; mais pour ce faire on est obligé de faire des hypothèses sur le profil de concentration dans le métal, par exemple supposer qu'il peut être représenté par une fonction erreur complémentaire de la profondeur. Cette décomposition se fait à partir de la région du spectre correspondant aux faibles énergies, sans pour autant faire d'hypothèses sur la valeur du coefficient de diffusion de l'élément dosé ( fig. 4). A partir du spectre ainsi décomposé, on peut d'une part mesurer l'épaisseur de la couche superficielle et d'autre part déterminer le profil de concentration de l'élément dosé dans le métal sous-jacent à la couche superficielle. On peut ainsi mesurer des couches d'oxydes dont l'épaisseur est comprise entre quelques $\AA$ et plusieurs milliers d'Å, d'autres méthodes d'extraction de profils de concentration seront présentées dans [16].

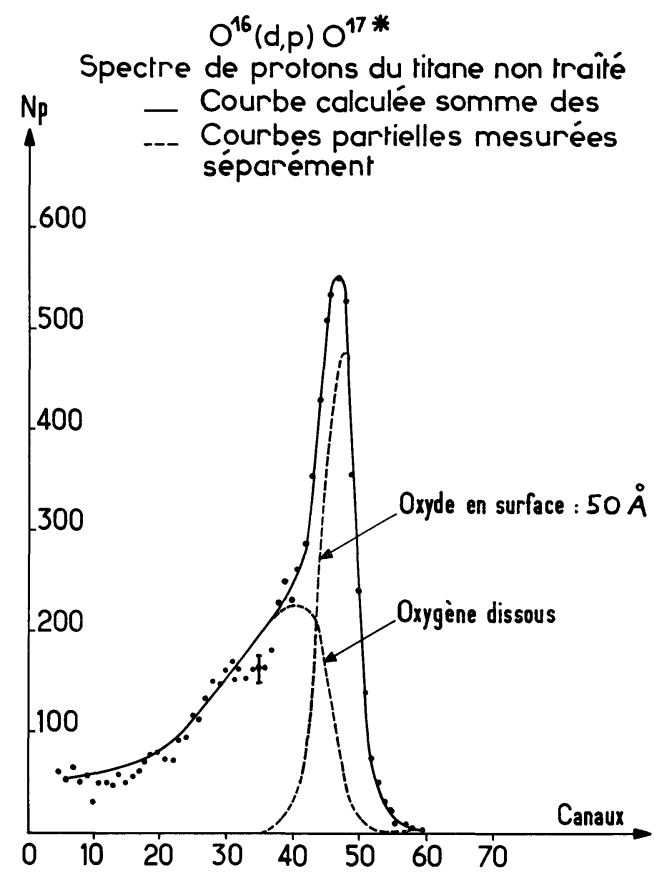

FIG. 4. - Décomposition d'un spectre correspondant à un échantillon de titane poli chimiquement. Le pic indique la présence d'une couche d'oxyde de $50 \AA$; le continuum provient de l'oxygène dissous dans le métal avant tout traitement.

b) Diffusion profonde. - Dans le cas d'une diffusion profonde (par exemple dosage de l'oxygène dans des échantillons de zirconium après oxydation à haute température), on est amené à faire des dosages après des abrasions ou des attaques chimiques successives de la surface de l'échantillon [20,21]. L'emploi des traceurs radioactifs pour l'étude des phénomènes de diffusion conduit souvent à recourir à cette méthode bien qu'elle soit destructive. En pratique, on ne peut utiliser la méthode des abrasions successives que dans la mesure où le gradient de concentration est suffisamment faible pour que l'on puisse considérer la concentration comme constante sur une profondeur de l'ordre du micron. Cependant, il peut arriver, dans certains cas, qu'une couche d'oxyde par exemple vienne se former spontanément à la surface (cas du zirconium), modifiant ainsi la forme du spectre [25]. On est alors amené à décomposer le spectre comme cela est indiqué dans le paragraphe précédent afin d'éliminer la contribution de la couche superficielle. Le nombre total de « coups » (particules détectées) correspondant à ce spectre (intégrale du spectre) est proportionnel au nombre d'atomes d'oxygène situés entre la surface de l'échantillon et une profondeur $X_{0}(2$ à $3 \mu \mathrm{m})$, les réactions ayant lieu à une profondeur supérieure n'étant pas détectées. La cote $X_{0}$ ainsi définie ne dépendant pas de la profondeur d'abrasion, l'intégrale du spectre représenté en fonction des épaisseurs enlevées donne donc directement l'allure de la courbe 
de concentration de l'élément léger à doser en fonction de la profondeur. La valeur absolue de la concentration est calculée en comparant l'échantillon à une couche mince de référence d'épaisseur connue.

DesGription de L'APPAREILlage. - Nous avons utilisé pour nos expériences l'accélérateur Van de Graaff de $2 \mathrm{MeV}$ de l'École Normale Supérieure de Paris. L'énergie est définie par la valeur du champ magnétique de l'aimant de déviation. La détection des particules chargées, produites au cours des réactions nucléaires envisagées a été réalisée à l'aide d'un détecteur à semiconducteur Ortec. Un film de Mylar placé devant le détecteur élimine les particules rétrodiffusées (diffusion élastique). L'angle solide de détection est de $3 \times 10^{-2}$ stéradian (détecteur de $3 \mathrm{~cm}^{2}$ placé à $10 \mathrm{~cm}$ de la cible), l'angle de détection étant de $165^{\circ}$ ( fig. 1). Ce choix de $165^{\circ}$ est un compromis :

- On a intérêt à avoir un angle de détection aussi voisin de $180^{\circ}$ que possible [10], [16].

- Il n'est pas possible d'avoir un angle de $180^{\circ}$, car cela signifierait que l'on intercepte le faisceau des particules incidentes, d'où le choix de $165^{\circ}$.

Les échantillons sont placés sous vide secondaire perpendiculairement au faisceau incident.

Les impulsions, après amplification et mise en forme, sont traitées par un analyseur Intertechnique à 400 canaux ( $f i g .1$ ). La durée d'un enregistrement est de l'ordre de quelques minutes.

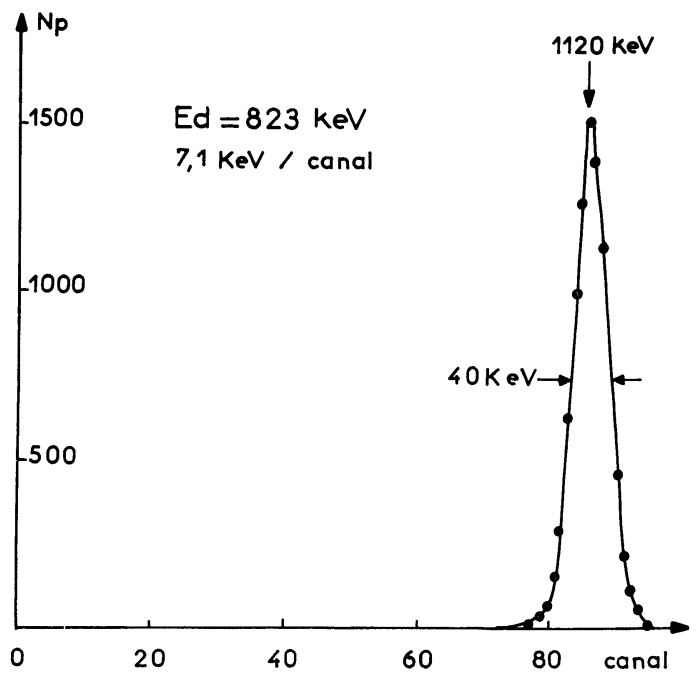

FIG. 5. - Courbe de résolution pour la réaction $\mathrm{O}^{16}(\mathrm{~d}, \mathrm{p}) \mathrm{O}^{17^{*}}$ enregistrée avec une cible de $\mathrm{Ta}_{2} \mathrm{O}_{5}$ de $160 \AA$ à $300 \mathrm{keV}$. La résolution équivalente en profondeur est de $0,25 \mu \mathrm{m}$.

La résolution de l'appareillage a été déterminée, dans le cas du zirconium, à l'aide d'une couche anodique d'oxyde de tantale (ox. à $10 \mathrm{~V}$ ), d'épaisseur

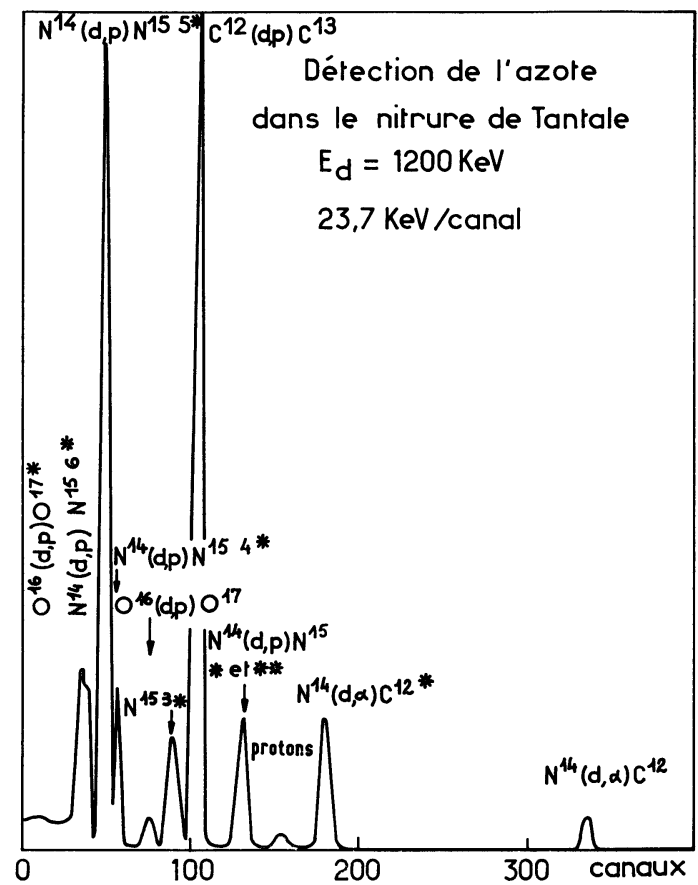

FIG. 6. - Spectre provenant d'une cible de nitrure de tantale, bombardée par des deutons. Les deux pics de rayon $\alpha$ vers les hautes énergies permettent de doser l'azote avec efficacité.

$160 \AA$ (fig. 5). La largeur à mi-hauteur du spectre obtenu étant de $40 \mathrm{keV}$, cela correspond à une résolution de $0,25 \mu \mathrm{m}$ pour le zirconium.

La figure 6 montre à titre d'illustration un spectre complexe correspondant au bombardement par des deutons d'une cible mince de nitrure de tantale. On voit que la réaction $\mathrm{N}^{14}(\mathrm{~d}, \alpha) \mathrm{C}^{12}$ qui fournit un rayon $\alpha$ est très énergique; ceci constitue un moyen efficace de détecter l'azote, comme on le verra.

IV. Applications à la métallurgie. - Nous avons utilisé cette méthode pour résoudre certains problèmes posés en métallurgie par le dosage des éléments légers. Nous allons donc décrire ces applications en nous limitant essentiellement à l'oxygène et à l'azote et nous décrirons une extension de la méthode au cas du carbone et du silicium.

1. Dosage de l'oxygène. - A. HÉtÉrodiffusion. a) Diffusion peu profonde et état de surface. - La méthode de microanalyse par observation des réactions nucléaires s'applique particulièrement bien à l'étude de la couche superficielle des métaux.

Nous l'avons appliquée à l'étude des échantillons de zirconium polis chimiquement dans un bain fluonitrique ( $\mathrm{HF} 5 \%, \mathrm{H}_{2} \mathrm{O} 35 \%, \mathrm{HNO}_{3} 60 \%$ ) [19]. L'examen du spectre obtenu ( fig. 7) permet de distinguer deux parties : un pic étroit dans la région des hautes énergies de protons, comparables au spectre 


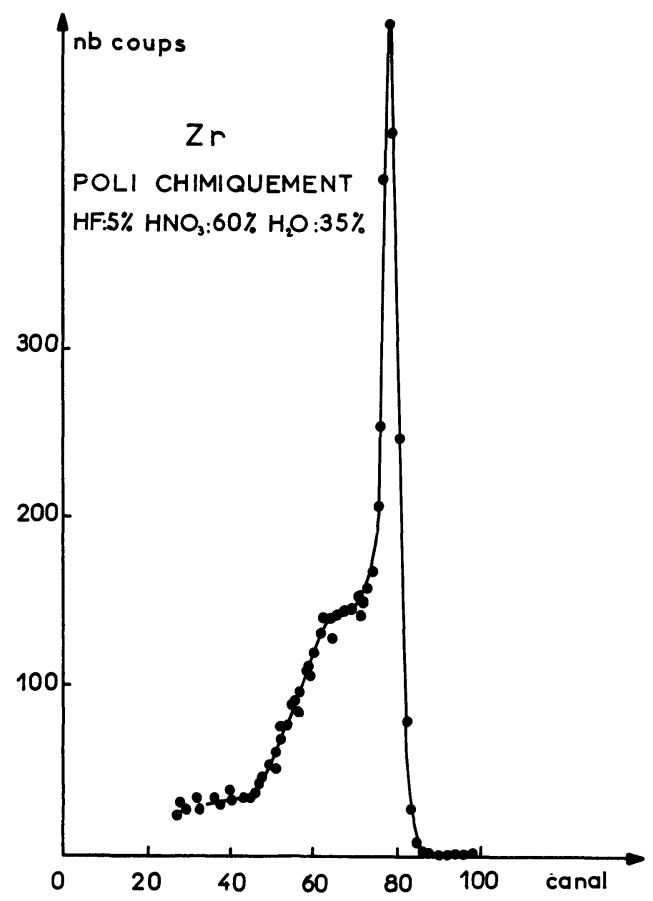

FIG. 7. - Spectre de protons enregistré sur un échantillon de zirconium poli chimiquement ( $\mathrm{HF} 5 \%$ \% $\mathrm{H}_{2} \mathrm{O} 35 \%$, $\mathrm{HNO}_{3} 60 \%$ ).

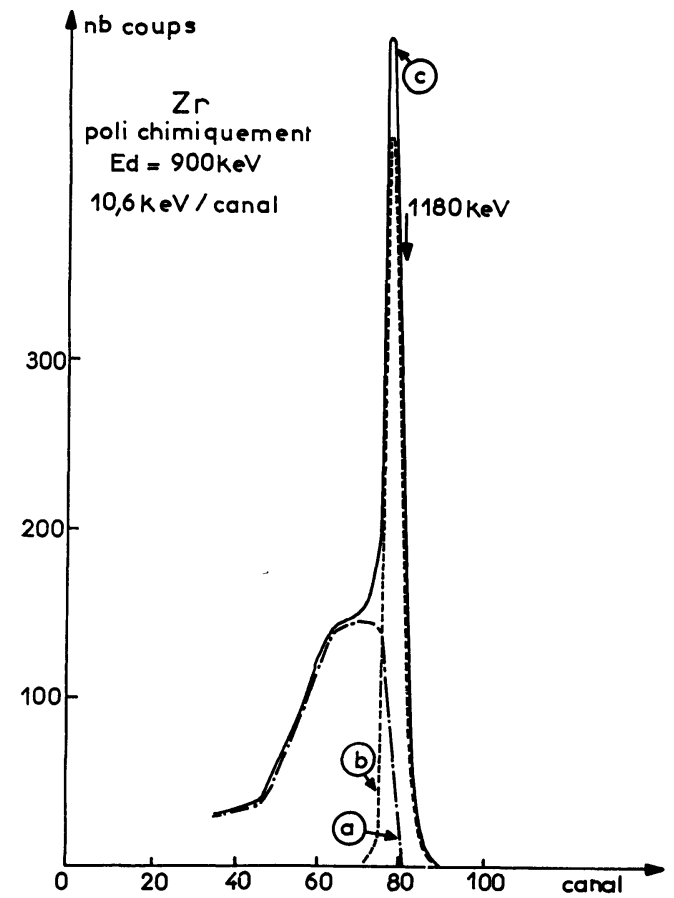

FIG. 8. - Décomposition du spectre de la figure 7 :
a) Oxygène dissous.
b) Pic d'oxyde superficiel.
c) Somme de a et $b$.

d'énergie donné par une couche mince d'oxyde de tantale ( $f g .5)$, et une autre partie dans la région des plus faibles énergies, correspondant à un nombre de protons plus faible. Nous avons interprété ce résultat [21] en supposant que les protons de haute énergie proviennent de la réaction des deutons incidents avec des atomes d'oxygène localisés dans une couche d'oxyde superficiel formée lors du polissage chimique et que les protons de faible énergie proviennent de la réaction avec des atomes d'oxygène en solution solide dans le zirconium. Le zirconium utilisé est en effet du zirconium élaboré par le procédé Kroll et une analyse chimique a montré qu'il contenait environ $600 \mathrm{ppm}$ d'oxygène. Pour préciser quantitativement cette interprétation, nous avons procédé à la décomposition du spectre ( fig. 8). Nous avons constaté, par comparaison avec le réseau de spectre calculé, que la forme de la partie du spectre expérimental relative aux faibles énergies était identique à celle donnée par une répartition uniforme d'oxygène, la concentration étant égale à 600 ppm, c'est-à-dire la teneur en oxygène initialement présente dans le métal. En retranchant du spectre expérimental la contribution de cette répartition uniforme d'oxygène, nous avons obtenu un pic présentant exactement la forme donnée par un oxyde mince superficiel. La comparaison du nombre de protons correspondant à cet oxyde avec celui des protons obtenus dans des conditions de bombardement analogues sur une couche de tantale formée par oxydation anodique nous a permis de déterminer le nombre d'atomes d'oxygène présents dans la couche mince de zircone, et par conséquent l'épaisseur de la couche considérée : $80 \pm 5 \AA$. Ainsi, après un tel polissage chimique, on observe la présence d'une mince couche superficielle d'oxyde sans dissolution supplémentaire d'oxygène dans le métal.

Nous avons également examiné l'influence d'un traitement thermique sous vide secondaire $\left(5 \times 10^{-6}\right.$ torr $)$ effectué après le polissage chimique de l'échantillon (fig. 9). La figure montre la comparaison de deux spectres obtenus respectivement sur un échantillon poli chimiquement et sur un échantillon poli chimiquement puis recuit sous vide secondaire 2 heures à $800^{\circ} \mathrm{C}$. A partir de ce qui a été dit ci-dessus, les conclusions suivantes peuvent être déduites :

- Le pic correspondant à l'oxyde superficiel montre que l'épaisseur de celui-ci n'a pas notablement varié après le recuit sous vide.

- La partie du spectre correspondant aux basses énergies traduit la présence d'une quantité plus importante d'oxygène dissous dans le zirconium après le recuit. La concentration d'oxygène de l'échantillon poli chimiquement est homogène et égale à 600 ppm; par contre, pour l'échantillon recuit sous vide, elle correspond à une diffusion d'oxygène sur une certaine profondeur. Les calculs théoriques ont permis de montrer que cette diffusion d'oxygène s'étendait jusqu'à une profondeur de l'ordre de $10 \mu \mathrm{m}$ et corres- 


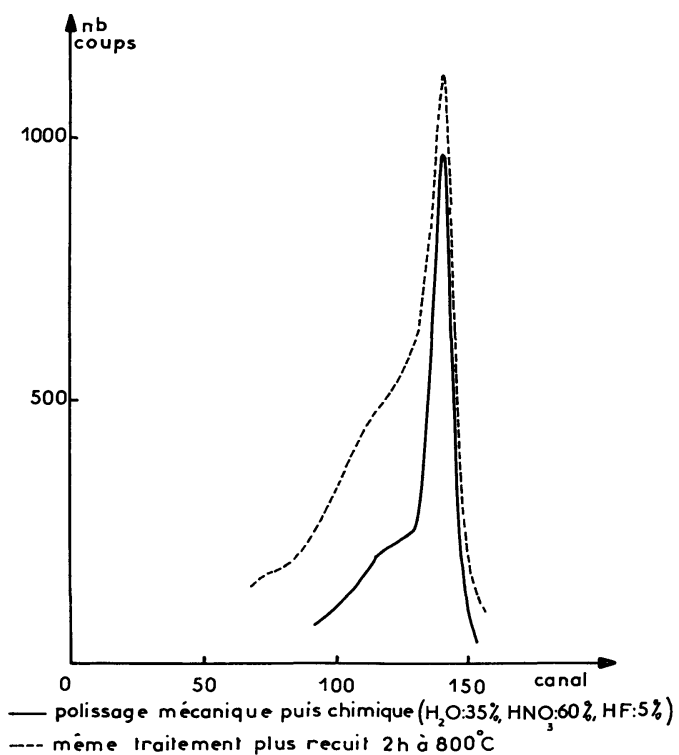

FIG. 9. - Influence d'un recuit sous vide poussé $\left(5 \times 10^{-6}\right.$ torr $)$ à $800^{\circ} \mathrm{C}$ pendant 2 heures sur un échantillon de zirconium : la quantité d'oxygène dissous a plus que doublé près de la surface ; la couche d'oxyde a subsisté.

pondait à une concentration de 1200 ppm d'oxygène dans le métal au voisinage de l'interface métal-oxyde. La quantité d'oxygène ayant ainsi diffusé est supérieure à celle initialement fixée sur l'échantillon par le polissage chimique. Des essais, toujours basés sur la méthode de microanalyse par observation des réactions nucléaires, sont actuellement en cours afin de préciser le mécanisme de formation de cette couche d'oxyde superficiel. Notons en outre que les résultats obtenus sur des échantillons d'alliage zirconium-cuivre polis chimiquement ou recuits sous vide sont analogues à ceux obtenus sur du zirconium pur [21].

b) Diffusion profonde. - Au cours de l'oxydation à haute température du zirconium ou de ses alliages [22], [23], il y a simultanément formation d'une couche superficielle d'oxyde et dissolution d'oxygène dans le métal sous-jacent pour former une solution solide interstitielle. Le problème qui se pose est de déterminer le profil de concentration en oxygène dissous en fonction de la profondeur pour des conditions d'oxydation données. Les essais ont été effectués sur des échantillons de zirconium Kroll et d'alliages zirconium-cuivre $2,5 \%$ en poids dont la teneur initiale en oxygène était d'environ $600 \mathrm{ppm}$.

Les échantillons de forme parallélépipédique $(2 \times 10$ $\times 15 \mathrm{~mm}$ ), après avoir été abrasés mécaniquement jusqu'au papier 600 (sous courant d'eau), ont été ensuite recuits pendant 2 heures à $800^{\circ} \mathrm{C}$ sous une pression de $10^{-6}$ torr. Après le traitement de recristallisation, chaque échantillon était repoli au papier 600 , soigneusement dégraissé à l'acétone, puis poli chimi- quement. Ce polissage était réalisé dans un bain fluonitrique de composition : $\mathrm{HF} 5 \%, \mathrm{H}_{2} \mathrm{O} 35 \%$, $\mathrm{HNO}_{3} 60 \%$; l'échantillon étant agité constamment pendant une durée totale d'une minute environ, le polissage était interrompu toutes les $15 \mathrm{~s}$ par un rinçage abondant sous l'eau courante. Après ce polissage, les échantillons étaient soigneusement rincés à l'alcool et à l'eau distillée avant d'être séchés. Ces préparations d'état de surface doivent être réalisées dans des conditions rigoureuses, car certains auteurs ont montré que l'état de surface initial du métal influe considérablement sur les résultats.

Les études (par utilisation de la réaction nucléaire $\mathrm{O}^{16}(\mathrm{~d}, \mathrm{p}) \mathrm{O}^{17^{*}}$ avec une énergie de bombardement de $900 \mathrm{keV}$ ) du profil de diffusion de l'oxygène dans le métal sous-jacent à l'oxyde ont porté sur les échantillons ayant subi les traitements d'oxydation suivants, ces traitements d'oxydation ayant été faits sous un courant d'oxygène pur, le débit étant d'un litre par heure environ :

— zirconium oxydé pendant 24 heures à $750^{\circ} \mathrm{C}$,

- zirconium-cuivre à $2,5 \%$ de cuivre oxydé 24 heures à $750^{\circ} \mathrm{C}$,

- zirconium oxydé pendant 450 heures à $750^{\circ} \mathrm{C}$,

- zirconium oxydé pendant 400 heures à $850^{\circ} \mathrm{C}$.

Dans ces deux derniers cas, les courbes de microdureté tracées sur ces échantillons ( fig. 10) présentaient nettement des paliers déjà mis en évidence par de

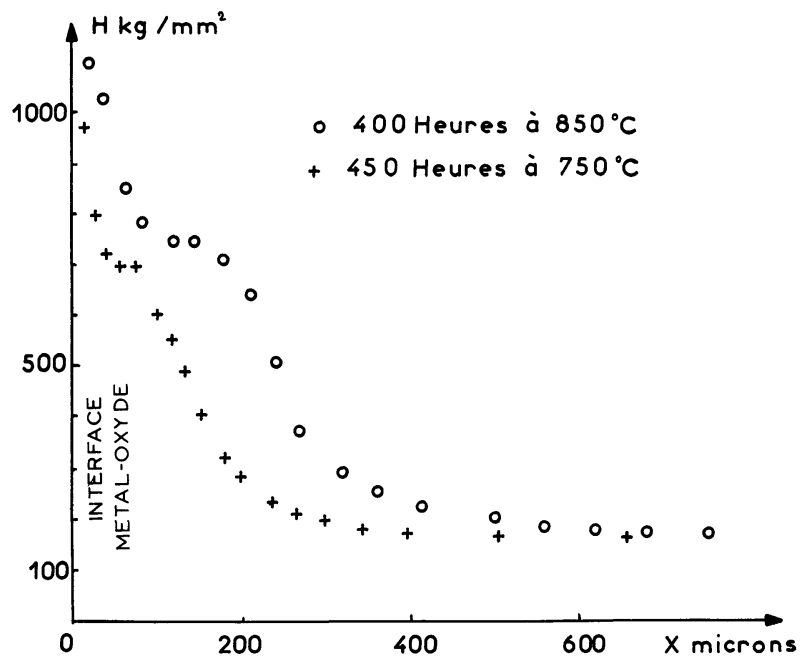

FIG. 10. - Courbes de microdureté en fonction de la profondeur dans un échantillon de zirconium ayant subi une diffusion profonde; on remarque le palier de dureté.

nombreux auteurs $[24,25,26]$. Nous désirions savoir si ces paliers de microdureté correspondaient ou non à des paliers de concentration en oxygène [27].

Après oxydation, chaque échantillon était abrasé mécaniquement à l'aide d'une machine à polir alternative qui permet de faire des abrasions successives 
parallèlement à un plan de référence (le plan de surface de l'échantillon). Les profondeurs atteintes étaient repérées par rapport à l'interface des échantillons, celles-ci étant déterminées par pesée des échantillons avant et après abrasion. La profondeur d'abrasion ainsi déterminée l'est à mieux de $2 \mu \mathrm{m}$ près.

Pour des raisons de commodité d'utilisation de l'accélérateur, au lieu d'abraser successivement le même échantillon pour faire les dosages, nous avons préféré abraser à des profondeurs différentes une série d'échantillons préparés et oxydés dans des conditions identiques.

Résultats. - Analyse des spectres : la figure 5 montre la courbe de résolution de l'appareillage obtenue sur une cible mince d'oxyde de tantale. Les figures 11 et 12

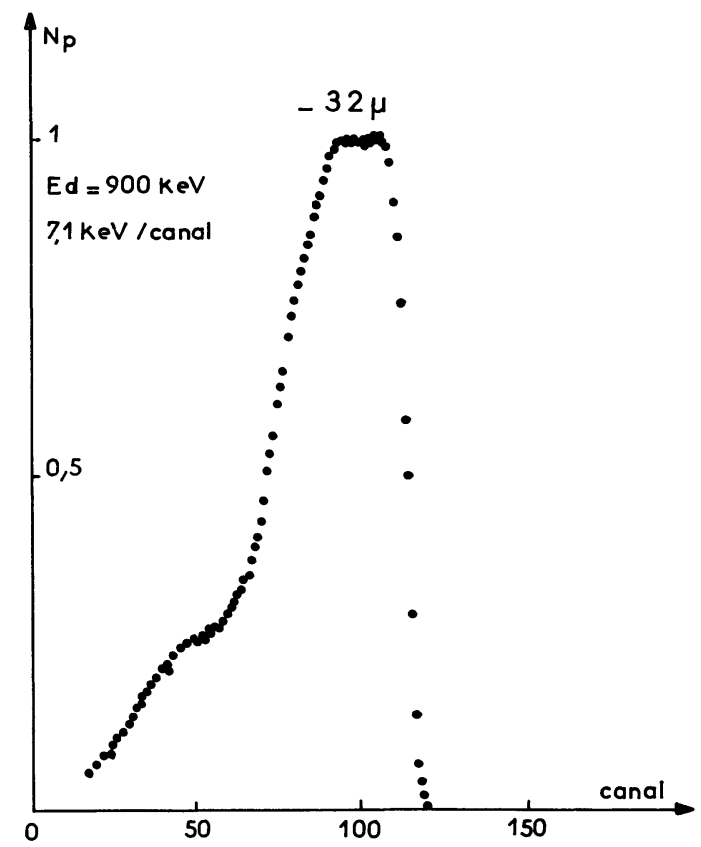

Fig. 11. - Spectre de protons correspondant à un échantillon de zirconium oxydé 24 heures à $750^{\circ} \mathrm{C}$ et abrasé à $32 \mu \mathrm{m}$ de l'interface oxyde-métal.

montrent les formes caractéristiques obtenues expérimentalement. On constate que la forme des spectres correspondant à des abrasions voisines de l'interface métal-oxyde est tout à fait comparable à celle obtenue sur des échantillons homogènes, ainsi qu'au spectre calculé ( $f i g .3$ ) correspondant à une concentration uniforme en oxygène dans l'échantillon. Cela signifie que la concentration en oxygène est trop lentement variable à l'intérieur de l'échantillon pour avoir une influence sur la forme du spectre. Cela justifie donc a posteriori la méthode de sectionnement utilisée pour effectuer ces dosages.

Les spectres correspondant à des profondeurs de pénétration plus importantes présentent tous un pic dans la région correspondant aux hautes énergies

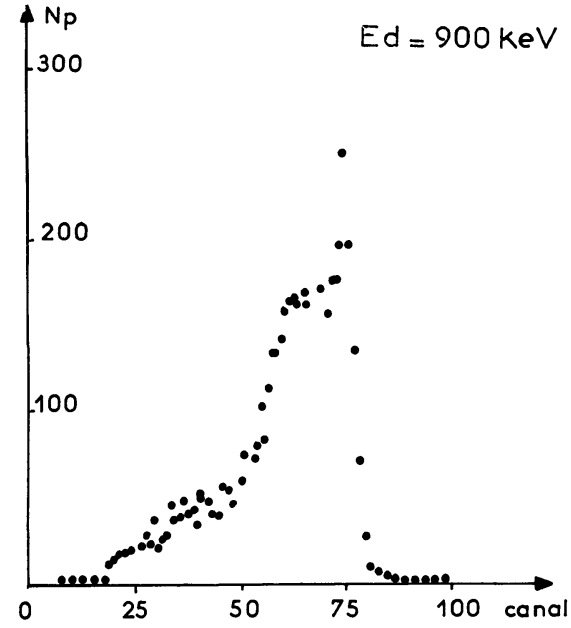

FIG. 12. - Spectre correspondant à une diffusion très profonde $\left(400 \mathrm{~h}\right.$ à $\left.850^{\circ} \mathrm{C}\right)$. Abrasion à $630 \mu \mathrm{m}$.

(fig. 12). Ce pic est dû à une mince couche d'oxyde formée à la surface lors du polissage chimique des échantillons au bain fluonitrique. Il est cependant facile de distinguer l'oxygène contenu dans la couche superficielle de l'oxygène en solution solide interstitielle dans le métal sous-jacent à l'oxyde.

Détermination quantitative de la concentration en oxygène. - La teneur en oxygène a été calculée en supposant que la concentration était uniforme dans chaque échantillon, cela étant justifié par le fait qu'il n'est pas possible de mettre en évidence des différences notables entre le spectre expérimental et le spectre calculé pour $\sqrt{D t}=\infty$. Dans tous les cas, la détermination de la quantité absolue d'oxygène contenu dans les échantillons a été faite, d'une part en reliant par le calcul les intégrales des spectres expérimentaux à l'intégrale du spectre obtenu par bombardement d'un échantillon mince d'oxyde de tantale $\mathrm{Ta}_{2} \mathrm{O}_{5}$ préparé par oxydation anodique à 55 volts (l'épaisseur de l'oxyde était de $880 \AA$ ), d'autre part par comparaison aux résultats obtenus sur des alliages zirconium-oxygène à concentration connue en oxygène, préparés au four à bombardement électronique. Nous avons pu constater que les écarts correspondant à ces diverses déterminations étaient inférieurs à $2 \%$ en valeur relative.

Nous avons tenté d'interpréter les courbes ainsi tracées en supposant qu'à partir d'une certaine distance de l'interface métal-oxyde $x_{1}$, le coefficient de diffusion $D$ de l'oxygène dans le zirconium est constant. A cet effet, nous avons utilisé la formule établie dans la référence [21] :

$$
\frac{C(x)-C_{0}}{C_{1}-C_{0}}=\frac{\operatorname{erfc} \frac{x+x_{0}}{2 \sqrt{D t}}}{\operatorname{erfc} \frac{x_{0}+x_{1}}{2 \sqrt{D t}}}
$$


où $x_{0}$ représente la distance de l'interface à la surface initiale du métal, $C_{0}$ la concentration initiale en oxygène du zirconium (600 ppm, soit $\left.3 \times 10^{20} \mathrm{at} / \mathrm{cm}^{3}\right)$, $C_{1}$ la concentration au point se trouvant à la profondeur $x_{1}$ par rapport à l'interface métal-oxyde. On constate sur les figures 13 et 14 , correspondant aux

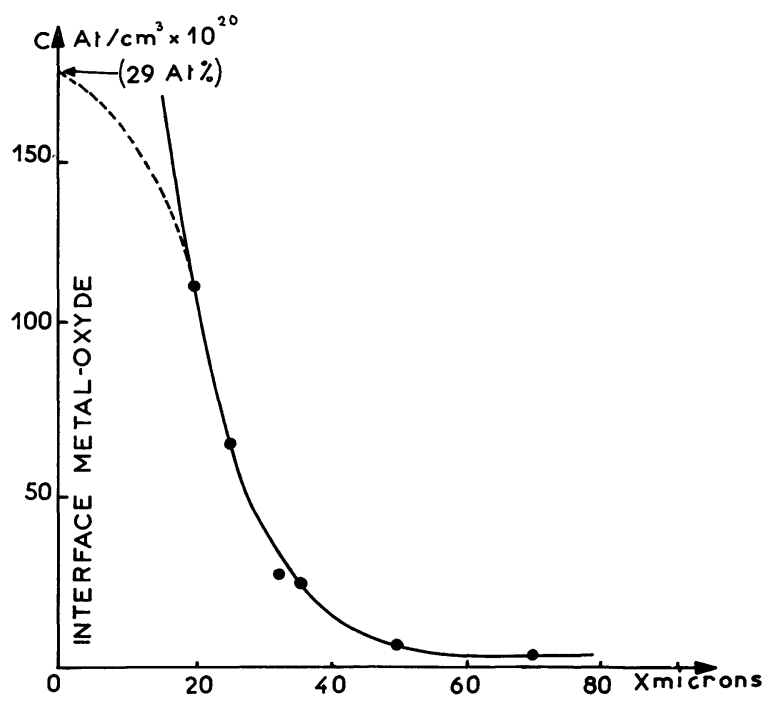

Fig. 13. - Profil de concentration de l'oxygène dans du zirconium oxydé 24 heures à $750^{\circ} \mathrm{C}$. La courbe en trait plein est un ajustement du type erfc.

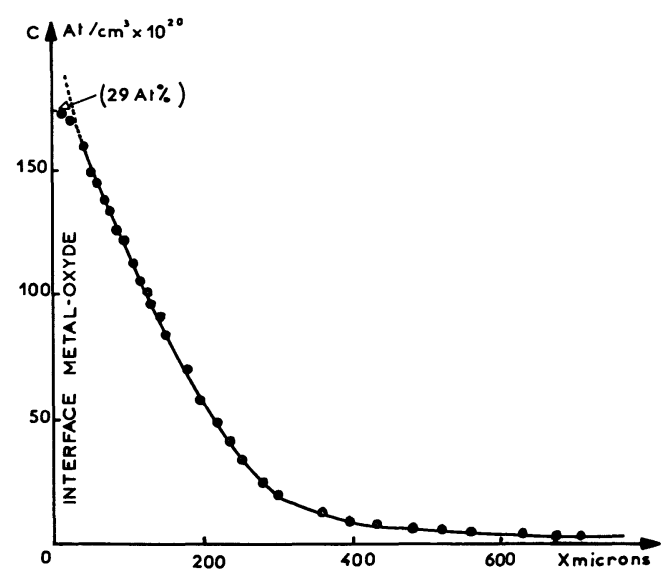

FIG. 14. - Profil de concentration de l'oxygène correspondant à l'échantillon de la figure 10 , oxydé $400 \mathrm{~h}$ à $850^{\circ} \mathrm{C}$. On voit que le palier de microdureté ne correspond à aucun accident du profil. La courbe en trait plein est un ajustement du type erfc.

traitements de 24 heures à $750^{\circ} \mathrm{C}$ et de 400 heures à $850^{\circ} \mathrm{C}$, que les points expérimentaux se placent à partir de $x_{1}$ avec une bonne précision sur des courbes en fonction erreur complémentaire. Pour ces deux courbes, les caractéristiques sont les suivantes :
Oxydation 24 heures A $750^{\circ} \mathrm{C}$

$$
\begin{aligned}
C_{0} & =3 \times 10^{20} \mathrm{at} / \mathrm{cm}^{3} \\
x_{1} & =20 \mu \\
C_{1} & =108 \times 10^{20} \mathrm{at} / \mathrm{cm}^{3} \\
D & =2,6 \times 10^{-11} \mathrm{~cm}^{2} / \mathrm{s}
\end{aligned}
$$

Dans tous les cas, on constate que, pour une profondeur inférieure à la profondeur $x_{1}$, il est impossible de faire correspondre la courbe expérimentale avec une fonction erreur complémentaire. On peut donc supposer que le coefficient de diffusion de l'oxygène dans le zirconium sous-jacent à l'oxyde augmente lorsque l'on approche de la concentration à saturation [21].

On constate de plus que, quel que soit le traitement d'oxydation, il n'y a pas de relation simple entre la dureté des échantillons et leur concentration en oxygène. Bien plus, dans le cas d'une oxydation de longue durée, le palier de microdureté que présentent les échantillons ne correspond pas à un accident quelconque de la courbe de concentration en oxygène. On remarque cependant que les valeurs de dureté mesurées par les divers auteurs sont sensiblement différentes [27], [28], [29]. Or les conditions d'oxydation, en particulier la durée et la température d'essai, varient d'une expérience à l'autre; ceci suggère que le traitement thermique a une influence notable sur la dureté de la solution solide $\mathrm{Zr}-\mathrm{O}$. Ainsi la dureté ne dépendrait pas seulement de la concentration en oxygène dissous, mais aussi des conditions du traitement thermique.

B. Autodiffusion. - Pour étudier l'autodiffusion de l'oxygène dans la zircone, nous avons réoxydé en atmosphère d'oxygène 18 dans des ampoules scellées des échantillons initialement oxydés en oxygène 16 . L'utilisation des réactions nucléaires $\mathrm{O}^{16}(\mathrm{~d}, \mathrm{p}) \mathrm{O}^{17^{*}}$ et $\mathrm{O}^{18}(\mathrm{p}, \alpha) \mathrm{N}^{15}$ permet de tracer les répartitions respec-

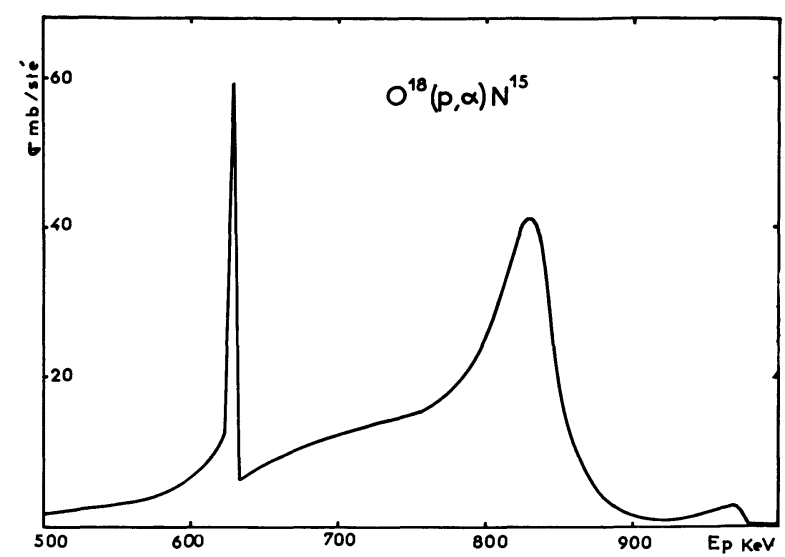

FIG. 15. - Section efficace différentielle à $165^{\circ}$ de la réaction $\mathrm{O}^{18}(\mathrm{p}, \alpha) \mathrm{N}^{15}$. 
tives de ces isotopes en fonction de la profondeur pour différentes conditions d'oxydation.

L'utilisation de la réaction $\mathrm{O}^{18}(\mathrm{p}, \alpha) \mathrm{N}^{15}$ présente pour le dosage de l'oxygène 18 un grand intérêt.

Comme il n'y a pas de réactions parasites et que le rendement est très grand, il en résulte une sensibilité extrêmement élevée : $5 \times 10^{13}$ atomes d'oxygène $/ \mathrm{cm}^{2}[11]$

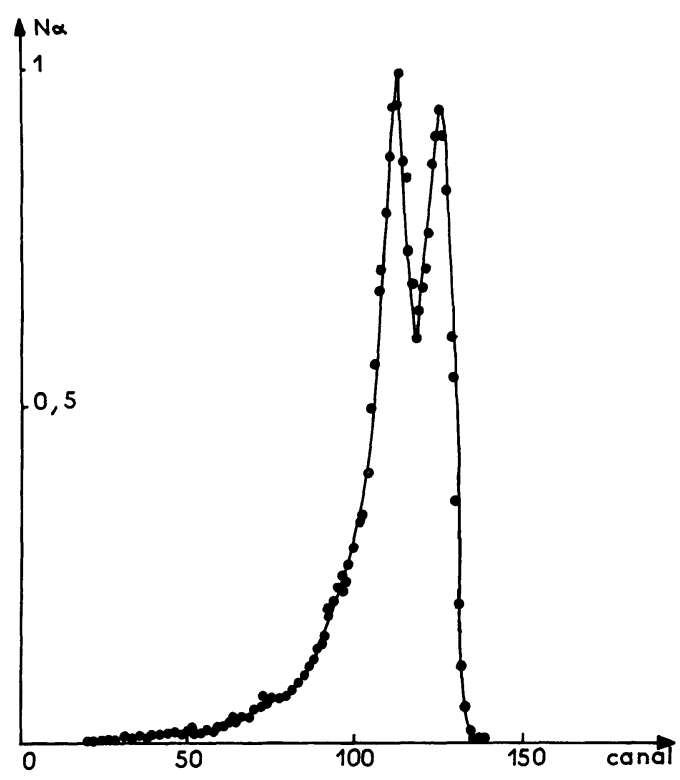

FIG. 16. - Spectre de rayons $\alpha$ d'un échantillon de zirconium oxydé dans $\mathrm{O}^{16}$ d'abord puis $\mathrm{O}^{18}$. Énergie des protons supérieure à celle de la résonance $(629 \mathrm{keV})$.

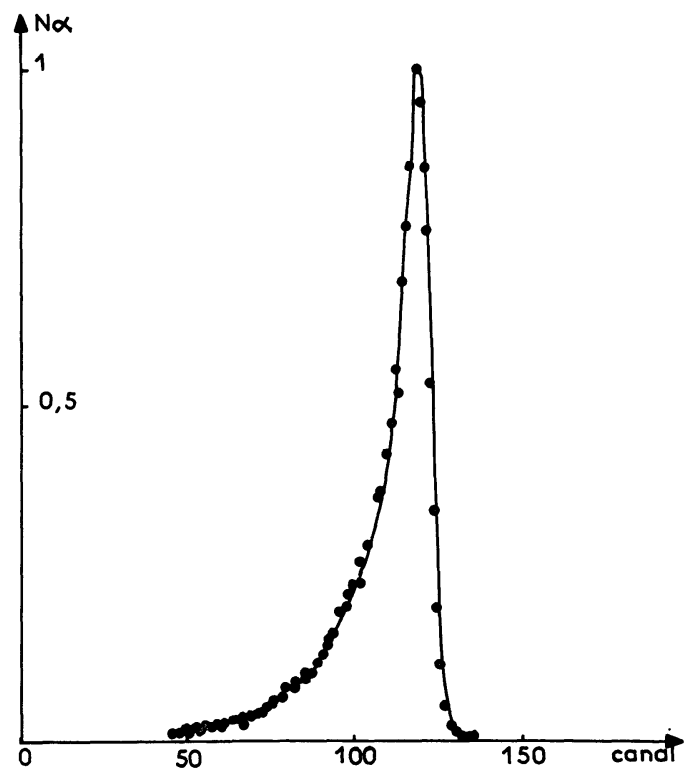

FIG. 17. - Même cas que la figure 16, mais l'énergie des protons étant inférieure à $629 \mathrm{keV}$, il n'y a plus qu'un pic. L'évolution de ces spectres en fonction de l'énergie permet une détermination très précise du profil de $\mathrm{O}^{18}$ dans la zircone.
On constate d'autre part que la courbe de section efficace (fig. 15) de cette réaction présente une résonance très étroite à $629 \mathrm{keV}$ dont la largeur est de $2,1 \mathrm{keV}$. Or, la courbe de section efficace a une grande influence sur la forme du spectre enregistré; on conçoit donc dans ce cas que celle-ci dépende beaucoup de l'énergie des protons incidents. Les figures 16 et 17 illustrent ce fait dans le cas de dosages effectués sur un échantillon de zirconium oxydé en atmosphère d'oxygène $\mathrm{O}^{16}$, puis recuit en atmosphère d'oxygène $\mathrm{O}^{18}$. On observe alors un spectre présentant soit deux pics, soit un seul pic, suivant que l'énergie de bombardement des protons est supérieure ou inférieure à l'énergie de résonance. Ceci permet un calcul encore plus précis du profil de diffusion.

En fait, nous disposons dans ce cas de deux possibilités de détermination du profil de concentration en $\mathrm{O}^{18}$ :

- une détermination directe à l'aide de la réaction $\mathrm{O}^{18}(\mathrm{p}, \alpha) \mathrm{N}^{15}$, comme nous l'avons vu précédemment,

- une détermination indirecte à l'aide de la réaction $\mathrm{O}^{16}(\mathrm{~d}, \mathrm{p}) \mathrm{O}^{17^{*}}$.

La figure 18 donne les spectres obtenus par la réaction $\mathrm{O}^{16}(\mathrm{~d}, \mathrm{p}) \mathrm{O}^{17^{*}}$ avec une énergie de bombar-

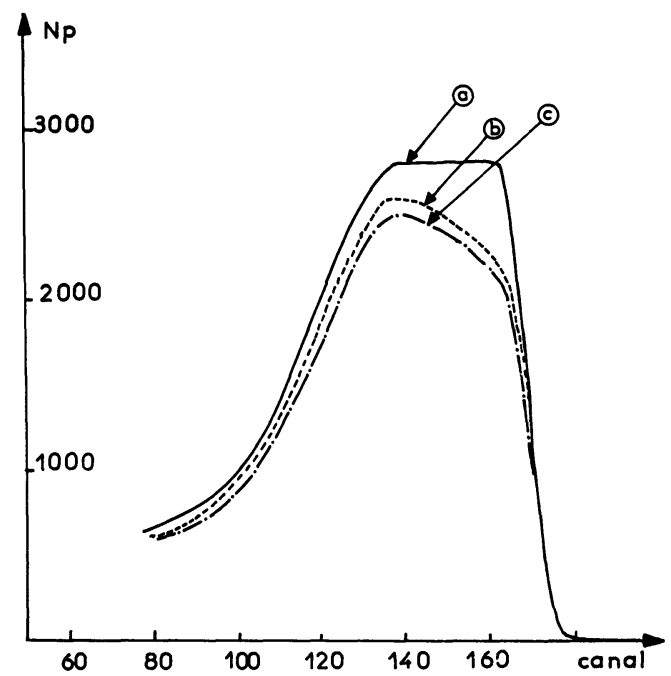

FIG. 18. - Spectre de protons enregistré pour des échantillons de même type que ceux des figures 16 et 17 , et indiquant le profil de $\mathrm{O}^{16}$ : a) zircone naturelle; b) et c) diffusions en atmosphère d'oxygène enrichi à $95 \%$ en $\mathrm{O}^{18} \cdot \mathrm{O}^{18}$ a partiellement remplacé $\mathrm{O}^{16}$ en surface.

dement de $900 \mathrm{keV}$ pour différents temps de recuit en atmosphère d'oxygène 18 . On constate que ce temps a une influence marquée sur la forme des spectres expérimentaux : en effet, l'oxygène 16 est remplacé par l'oxygène 18 et fait défaut près de la surface. 
La présence d'une résonance étroite de la réaction $\mathrm{O}^{18}(\mathrm{p}, \alpha) \mathrm{N}^{15}$ donne une résolution environ 10 fois meilleure que celle obtenue dans le cas du dosage de l'oxygène $16[9,10,16]$. Pour pouvoir tracer le profil de diffusion de l'oxygène 18 avec la précision maximale, on peut utiliser un analyseur bidimensionnel : en modifiant par sauts d'amplitude donnée l'énergie des particules incidentes, on modifie la profondeur à laquelle se produit la réaction étudiée correspondant à l'énergie de résonance et on obtient ainsi un spectre (fig. 19) très riche en information sur le profil de

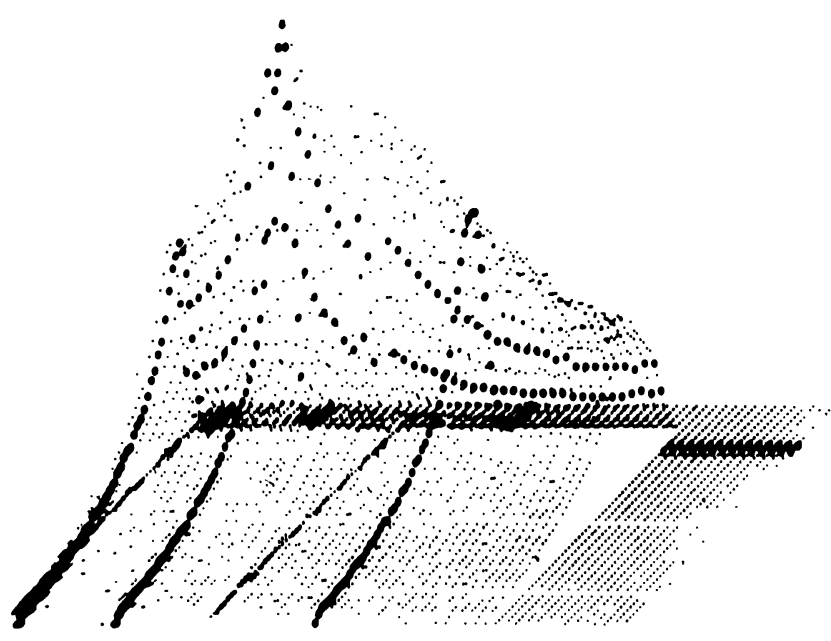

FIG. 19. - Spectre bidimensionnel de rayons $\alpha$ correspondant à une série de spectres du type de la figure 17, enregistrés à des énergies de bombardement $E_{\mathrm{p}}$ croissantes (horizontalement $1 \mathrm{keV} /$ canal). On distingue des coupes à $E_{\mathrm{p}}=$ Cte et d'autres à $E_{\alpha}=$ Cte.

concentration en $\mathrm{O}^{18}$. Ces mesures complétées par les mesures des teneurs résiduelles en oxygène 16 et oxygène 18 dans l'ampoule scellée qui a été utilisée pour la seconde oxydation permettent de plus d'atteindre le coefficient d'échange isotopique. Il nous est impossible actuellement de donner des résultats précis, les manipulations n'étant pas encore complètement exploitées.

2. Dosage de l'azote. - La méthode de dosage par observation des réactions nucléaires permet également de doser avec une bonne précision l'azote 14 grâce aux réactions $\mathrm{N}^{14}(\mathrm{~d}, \alpha) \mathrm{C}^{12}$ et $\mathrm{N}^{14}(\mathrm{~d}, \mathrm{p}) \mathrm{N}^{15}$. Le dosage de l'azote 15 est également possible, si bien que l'on peut envisager d'étendre à l'azote les problèmes envisagés dans le cas de l'oxygène avec le dosage des isotopes $\mathrm{O}^{16}$ et $\mathrm{O}^{18}$ (étude de la diffusion de l'azote dans les nitrures, détermination de la provenance de l'azote introduit dans les métaux...).

Cette méthode a été appliquée entre autres à l'étude de nitrures formés par traitements thermiques dans différentes atmosphères. Des observations ont été faites sur des échantillons de zirconium nitrurés en atmo- sphère d'oxygène et d'azote, avec des pressions partielles d'azote variables. A partir des spectres d'énergie enregistrés sur l'analyseur multicanaux, nous avons pu tirer les conclusions suivantes :

Les échantillons de zirconium traités à $788^{\circ} \mathrm{C}$ sous courant d'air de $5 \mathrm{mn}$ à $14 \mathrm{~h}$ s'oxydent fortement, mais il est pratiquement impossible de mettre en évidence une nitruration, quelle que soit la profondeur par rapport à l'interface métal-oxyde à laquelle se fait le dosage. On peut montrer par comparaison à des cibles d'épaisseur connue de nitrure de tantale qu'il y a environ 2500 fois moins d'atomes d'azote que d'atomes d'oxygène dans la couche formée à la surface d'un échantillon traité 14 heures à $788^{\circ} \mathrm{C}$.

Par contre, pour des pressions partielles d'azote beaucoup plus faibles, il est possible de mettre en évidence une certaine nitruration des échantillons de zirconium. Ainsi, pour des échantillons de zirconium recuits 24 heures à $7500^{\circ} \mathrm{C}$ dans une atmosphère d'oxygène 18 contenant $8 \%$ d'azote 14 en volume - le traitement étant effectué en ampoule scellée, avec une pression initiale de $95 \mathrm{~g} / \mathrm{cm}^{2}$ - le zirconium a fixé environ 25 fois moins d'azote que d'oxygène.

Pour le premier traitement thermique, il y a eu fixation de $4 \times 10^{15}$ atomes d'azote par $\mathrm{cm}^{2}$, alors que pour le second traitement thermique il y a eu fixation de $115 \times 10^{15}$ atomes $/ \mathrm{cm}^{2}$.

L'interprétation des résultats énoncés ci-dessus est rendue délicate car les expériences ne sont pas directement comparables. Il semble cependant très net que la pression partielle de l'azote ait un rôle important au cours des traitements de nitruration.

Quelques mesures ont été effectuées sur des échantillons traités à $800^{\circ} \mathrm{C}$ en atmosphère d'ammoniac. Dans de telles conditions, on a pu mettre en évidence que le zirconium, le tantale, le titane étaient très avides d'azote.

Sur du silicium, par contre, on a pu déceler la formation d'un film mince de nitrure (quelques couches moléculaires d'épaisseur), mais nous n'avons pu mettre en évidence de diffusion d'azote dans le métalloïde sous-jacent.

Le nickel, quant à lui, est pratiquement insensible à l'action du gaz ammoniac.

3. Dosage d'autres éléments. - Nous avons vu dans un paragraphe précédent qu'en bombardant un échantillon de zirconium avec un faisceau de deutons (énergie de bombardement de $900 \mathrm{keV}$ ), il était possible de doser l'oxygène contenu dans l'échantillon. Or ce dosage (de 500 à $1200 \mathrm{keV}$ ) est obtenu en n'analysant qu'une bande étroite des énergies des particules émises au cours de l'expérience. On peut, en étendant cette bande d'énergie, doser plusieurs autres éléments en même temps que l'oxygène, en particulier le carbone et le silicium. Nous avons appliqué cette propriété à l'étude de l'influence de la 
préparation mécanique des échantillons sur les incorporations d'éléments à leur surface [19].

Les différents modes de préparation entraînent, en plus des perturbations mécaniques, une contamination importante de la couche superficielle ainsi que le

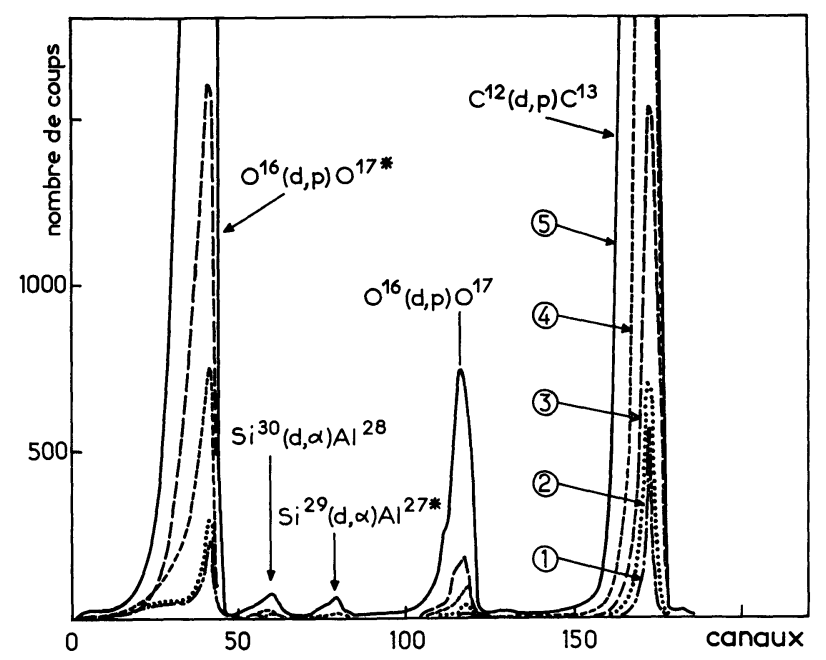

FIG. 20. - Spectres complexes enregistrés sur des échantillons de zirconium ayant subi divers traitements de polissage et indiquant l'incorporation de noyaux de $\mathrm{O}^{16}$, $\mathrm{Si}$ et $\mathrm{C}^{12}: 1$, polissage chimique ; 2 , alumine ; 3 , carbure de silicium humide ; 4 , diamant ; 5 , carbure de silicium sec.

montrent les spectres reportés sur la figure 20. Nous pouvons tirer les conclusions suivantes :

Le zirconium poli au papier abrasif au carbure de silicium à sec contient en surface une quantité d'oxygène maximale, alors que les échantillons polis à l'alumine ou chimiquement en contiennent environ 35 fois moins. Des constatations analogues peuvent être faites en ce qui concerne le carbone. Le polissage au diamant introduit des quantités très importantes de carbone, mais le rapport des quantités de carbone introduites lorsque l'on passe d'un mode de polissage à l'autre varie moins qu'en ce qui concerne l'oxygène : 1 à 15 au lieu de 1 à 35 .

Les inclusions de silicium sont pratiquement équivalentes et relativement faibles quel que soit le mode de polissage utilisé, sauf dans le cas du polissage au papier carbure à sec.

La rugosité au voisinage de la surface résultant du polissage mécanique provoque un élargissement des spectres par rapport à ceux obtenus sur une surface rigoureusement plane : il n'est alors possible de déduire que des informations sur la profondeur moyenne intéressée par les traitements de surface.

D'après ces résultats, nous constatons que deux types de polissage seulement permettent d'éviter convenablement les incorporations d'éléments étrangers : le polissage à l'alumine et le polissage chimique. Cependant, il faut noter que le polissage à l'alumine sur feutre entraîne des vallonnements caractéristiques à la surface de l'échantillon. Ainsi le polissage chimique constituerait la meilleure méthode de préparation d'état de surface du zirconium du point de vue à la fois microgéométrique et chimique.

V. Conclusions. - Nous avons pu montrer que l'utilisation de la méthode de microanalyse par observation des réactions nucléaires permet de déterminer non seulement les divers éléments présents au voisinage immédiat de la surface des échantillons (sur une profondeur de 2 à $3 \mu \mathrm{m}$ ), mais encore, par combinaison avec la méthode de sectionnement pour les diffusions profondes, de tracer des profils de concentration d'un élément dans un substrat métallique ou non.

L'énergie des particules détectées est spécifique du noyau bombardé, ce qui permet le dosage simultané de plusieurs éléments, par exemple oxygène, azote, silicium...

Cette méthode présente de nombreux avantages par rapport aux autres méthodes classiques de dosage, notamment par sa sensibilité, sa précision, sa rapidité. De plus, elle est non destructive et permet de séparer et de doser les différents isotopes d'un même élément.

Cette méthode donne pour les mesures absolues de concentration (concentration d'oxygène, d'azote ou d'autres éléments) une erreur de l'ordre de $1 \%$. La précision dans le cas d'une étude de diffusion profonde est donc équivalente à l'erreur commise sur la détermination de la profondeur d'abrasion.

Les limites de mesure des épaisseurs des couches d'oxydes, de nitrures, de siliciures... sont assez larges : de quelques $\AA$ jusqu'à 2 à $3 \mu$, avec une précision de $2 \%$ environ. La méthode présente en outre l'avantage de distinguer les atomes qui se trouvent dans la couche superficielle de ceux dissous dans le métal sous-jacent, sans pour autant faire aucune hypothèse sur la valeur du coefficient de diffusion de l'élément dans le métal.

Cette méthode dont nous avons illustré les possibilités essentiellement dans le cas du zirconium et de ses alliages peut s'appliquer à d'autres métaux : seules seront modifiées par rapport au cas du zirconium les relations parcours-énergie des particules dans le métal.

La méthode que nous avons décrite ci-dessus et illustrée dans le cas de certaines applications métallurgiques présente donc les avantages suivants :

- faible énergie de bombardement des particules, deutons ou protons permettant d'utiliser des accélérateurs de moins de $2 \mathrm{MeV}$,

- absence de fond continu naturel et rendement élevé des particules chargées entraînant une haute sensibilité d'analyse,

- dosage des éléments légers et des différents isotopes d'un même élément,

- haute spécificité des réactions employées,

- souplesse d'expérimentation, en particulier temps assez court d'analyse, quelques minutes usuellement,

- analyse en surface ou près de la surface. 
Cette méthode s'applique particulièrement bien à l'analyse de nombreux éléments légers dans les solides au voisinage de la surface, analyse qui ne peut pas toujours être menée à bien par les méthodes plus classiques, mais également dans le cas d'autres disciplines comme la botanique, la biologie [11]...

\section{BIBLIOGRAPHIE}

[1] Guinier (A.) et Castaing (R.), C. R. Acad. Sci., 1951, 232, 1948.

[2] Castaing (R.), Thèse, Paris, 1951 ; Onera, 55, 1952.

[3] Albert (Ph.), EingelmanN (Ch.), May (S.) et PETIT (J.), Analyse par activation d'oxygène, de carbone et d'azote au moyen de la réaction $(\gamma, \mathrm{n})$, C. R. Acad. Sci., 1962, 254, 119.

[4] Holi (J. B.) et CondiT (R. H.), U.C.R.L., 7938 (1965) ; C.A. 63, 9140 ; J. Electrochem. Soc., 1964, 111, 1132.

[5] DÖRFFLER (W. W.), Proceedings of IAEA, Symposium on "Thermodynamics", Vienne, 1966, p. 373.

[6] Ligenza (J. R.) et Spitzen (W. G.), Phys. Chem. Solids, 1960, 14, 131.

[7] Castaing (R.) et Srodzian (G.), C. R. Acad. Sci., 1962, 255, 1893.

[8] Slodzian (G.), Thèse, Orsay, 1963 (Annales de Physique).

[9] Amser (G.) et Samuer, (D.), Phys. Chem. Sol., 1962, 23, 1707.

Amsel, (G.), L.A.L., Orsay, Rept no 1053, mai 1963.

[10] NadaI (J. P.), Thèse, Orsay, 1967.

[11] Amser (G.) et Samuer, (D.), Analytical Chemistry, 1967, 39, 1689.

[12] PALMER (D. W.), Nuclear Instruments, 1965, 38, 187.

[13] OlleRhead (R. W.), Almouist (E.) et KUEHNER (J. A.), J. Appl. Phys., 1966, 37, 2440.

[14] Cox (B.) et Rox (C.), Electrochem. Techn., 1966, 4, 121.

[15] Möll_ER (E.) et STARfeit (N.), Nucl. Inst., 1967, $50,225$.
[16] Amsel (G.), Nadai (J. P.) et D'ARTemare (E.), article général à paraître.

[17] Whaling (W.), Handbuch der Physik, 1958, 34, 215.

[18] Wirliamson (W.), Boujot (J. P.) et Picard (J.), Rapport C.E.A., R 3042.

[19] Amsel (G.), David (D.), Béranger (G.), Boisot (P.), DE Gelas (B.) et LACOMBE (P.), Communication au IIe Colloque Cefracor-Irsid.

[20] Choudhury (A.), Palmer (D.) et al., Solid State Comm., 1965, 3, 119.

[21] Amsel, (G.), Béranger (G.), DE Geias (B.) et Lacombe (P.), J. Appl. Phys., avril 1968, 39, 2246.

[22] Whitwham (D.), Paidassi (J.) et Herenguei (J.), dans "L'oxydation des métaux " de BÉNARD (J.), Gauthier-Villars, Paris, 1964, tome II, chapitre X.

[23] Gulbransen (F. A.) et ANDREW (F. K.), J. of Metals, 1957, 9, 394.

[24] BÉranger (G.), Thèse, Paris, 1967.

[25] Darras (R.), Lorriers (H.) et BACQUe (P.), J. Mat. Nucl., 1965, 17, 79.

[26] WAliwork (G. R.), SMeltzeR (W. W.) et Rosa (C. A.), Acta Met., 1964, 12, 409.

[27] Amsel (G.), Béranger (G.), Boisot (P.), David (D.), DE Geras (B.) et LaCombe (P.), C. R. Acad. Sci., note présentée le 10 juin 1968 .

[28] Debuigne (J.), Thèse, Paris, 1966, Métaux, Corrosion, Industrie, 1967, 43, 89, 186, 235.

[29] ERICSSON (T.), OSTBERG (G.) et LEHTINEN (B.), J. Nucl. Mat., 1968, 25, 322. 